\title{
Implementation of the vehicle recognition systems using wireless magnetic sensors
}

\author{
SERCAN VANÇIN and EBUBEKIR ERDEM* \\ Department of Computer Engineering, Firat University, 23100 Elazig, Turkey \\ e-mail: svancin@ firat.edu.tr; aberdem@ firat.edu.tr
}

MS received 23 May 2016; revised 26 October 2016; accepted 23 November 2016

\begin{abstract}
Wireless network sensors and their use in traffic monitoring, traffic density determination or vehicle speed detection and classification have recently been the focus of interest for researchers. This article describes how a new sensor circuit was designed to deliver instantaneous, real-time and novel solutions as a vehicle detection system, which is more powerful than the nodes used in other studies, and gives results with smaller error margins due to its serial communication qualification. With the proposed logic algorithm, it was possible to categorise the instantaneous traffic status of a road in four levels: no traffic, mild traffic, heavy traffic and very heavy traffic. Additionally, with the nodes placed at the beginning and the end of the road, the number of vehicles per hour for a day was determined and traffic was analysed. Then, vehicles passing by were classified with a proposed classification algorithm and magnetic signature length (MSL) parameter as cars, minibuses, buses and trucks, and an accuracy rate of $95 \%$ was obtained. As the last application, the direction of motion of the vehicle on the $x$-axis as well as left-to-right or right-to-left directions was determined, and the result was $94 \%$ accurate. The simplicity of the proposed algorithms, the absence of any complex mathematical calculations, the low cost of the sensor node and circuit and the low power consumption of the communication system demonstrate the superiority of this system in comparison with other studies.
\end{abstract}

Keywords. Wireless sensor networks; magnetic sensor; traffic congestion; vehicle detection; magnetic signature length.

\section{Introduction}

Recently, several people face transportation problems and traffic jams. In order to monitor traffic, collect information on traffic status and transmit this information to drivers, intelligent transportation systems (ITS) were designed [1]. These systems operate by counting the number of vehicles on the road and determining the speed of the vehicles or by acquiring images of the vehicles through video cameras. As a result, traffic jams can be reduced by traffic information analysis or traffic forecast [2].

Many studies report the development of vehicle identification system by wireless sensor networks. Traffic conditions of the roads have been examined using anisotropic magnetic sensors (AMRs) and microphone sensors [3]. In addition to these, acoustic sensors [4], ultrasonic sensors [5] and video camera analysis systems or aerial images [6] have been used as a part of vehicle identification with wireless sensor network technology. In another study, magnetometer sensors were used [7]; magnetic fields detected above a threshold were measured in time as signals identifying the vehicles and thus compass applications

*For correspondence were developed. Yet another study designed a real-time traffic control system using wireless sensor networks [8]. In another study, a traffic monitoring system integrated with mobile data imaging and, as a result, management was suggested [9]. Vehicle identification was made using wireless sensor networks with multiple sensors and light, middleweight and heavy vehicles were classified [10]. To classify the vehicles on the road, an optimally divided sample-based classification and regression tree algorithm (CART) was suggested in a study by Haijian et al [11]. A neural networks-based vehicle motion-mode identification method was proposed [12]. In a study conducted by Varaiya and his students [13], magnetic data in $z$-axis were determined as the minimum and maximum and threshold values were detected for traffic surveillance using wireless magnetic sensors.

In this study, sensor nodes that can operate in a multipurpose way were designed and the sensor circuits were built by implementing magnetic sensors (HMC5983L) on those nodes. The sensor nodes were designed to be cheaper than the other nodes on the market, such as TelosB or MicaZ. With the help of those sensor circuits, three different applications were developed. The first of these applications was the real-time detection of the four-level 
instantaneous traffic information from a single-lane road using multiple sensor nodes. These levels were no traffic, mild traffic, heavy traffic and very heavy traffic. The relevant traffic density was obtained with the suggested logic value finding algorithm. Among the greatest advantages of this method when compared to others $[3,11]$ were the use of multiple sensor nodes, the minimisation of analog data measurement errors through serial communication of the nodes, low-cost system components and high performance. Additionally, with the help of a node that is placed at the end of the road (Node 3), a 24-h condition of traffic was observed hourly as a part of this application. In the second application, the vehicles passing by were classified as cars, minibuses, buses and trucks. In previous studies, pattern recognition using inductive loop detectors [14] and valley-and-hill pattern models using magnetic sensors $[15,16]$ was suggested for vehicle classification. In this study, however, a magnetic signature length (MSL) concept was defined and suggested as a novel solution to the classification problem. MSL presented excellent solutions to the vehicle classification problem with the developed algorithm (classification algorithm) and made it possible to obtain more accurate results than the ones in the study by Haijian et al [11]. In the Haijian et al [11] study, vehicles were only classified as automobiles or buses; but in the present study, classification was made in four categories and with a short-time decision mechanism. Finally, direction of motion in the $x$-axis was determined for vehicles with the aforementioned sensor circuits. The use of only two sensor nodes and the high performance of the invented direction determination algorithm show that this study is superior to the previous works. The percentage accuracy was examined in a setting where 50 vehicles made pass by the road, from left to right and from right to left. It was seen that this study gave more accurate results than the previous study [10]. On the other hand, construction of the system with low-cost hardware devices was possible due to the structure of the designed wireless sensor network. This wireless sensor network has its superiority in the programming simplicity of the magnetometer and the sensor nodes used and in the transmission of the necessary information to the coordinator node in a star topology. Sensor nodes and magnetometer were connected in series communication to avoid complicated computations and obtain more accurate information. In order to avoid traffic jams, the acquired informationsuch as the traffic condition, vehicle type or direction of motion-could be sent instantaneously over the Internet to drivers who wished to enter the road under study.

The remainder of this paper is organised as follows. Section 2 summarises the topic of wireless sensor networks. Section 3 details the topic of magnetic sensors and the realisation of the system with an explanation of the thought scenario. Section 4 presents the experimental applications and system analysis are presented in three subsections. Lastly, section 5 discusses the results of this study and gives suggestions.

\section{Wireless sensor networks}

Wireless sensor networks are scattered network structures in which many sensors or sensor nodes are in wireless communication. Wireless communication occurs between a receiver and a transmitter without cable connections via light or electromagnetic waves. Sensor networks built with small devices are cheap and have the ability of self-organisation, which makes the sensor intercommunications easier. Sensors, also called detectors and probes, are the sensing elements in electronic applications. The main components of a sensor node are the microcontroller, receiver-transmitter, power supply, memory and one or more sensor components. Various node types are on the market, such as TelosA, TelosB, Mica2, MicaZ, eMote, IMote 2 and Sensenode, all of which can be used in wireless sensor networks [16-18].

A sensor has the ability to detect many physical quantities such as length, amount, area, mass flow, heat transfer, force, temperature, voltage, current, resistance, oxidation/reduction, flux density, condensation, content, magnetic moment and magnetic field. Those different sensors can be used in many different fields according to their uses $[19,20]$. For example, heat transfer or temperature measurements can be used to work against forest fires, whereas measurements with moisture, temperature or pressure sensors can be used in weather forecasting and so forth. To measure the magnetic field of the Earth, to identify a certain material or to determine the metallic properties of objects, anisotropic magnetoresistive sensors (AMRs) are used [21-23]. Because metals such as iron, nickel or cobalt affect the background magnetic field, many objects containing metals can be detected by these sensors. Because vehicles contain various metal parts as well, they can be detected using magnetic sensors. Similarly, traffic information on a road can also be obtained [24-26].

\section{Magnetic sensors and experimental system setup}

Magnetic sensors have been used for a long time. Although the early works were on basic direction finding and navigation, nowadays these applications have expanded. Magnetic sensors that can make more precise and more accurate measurements are now designed to work conveniently with integrated circuits. They have become more advantageous 


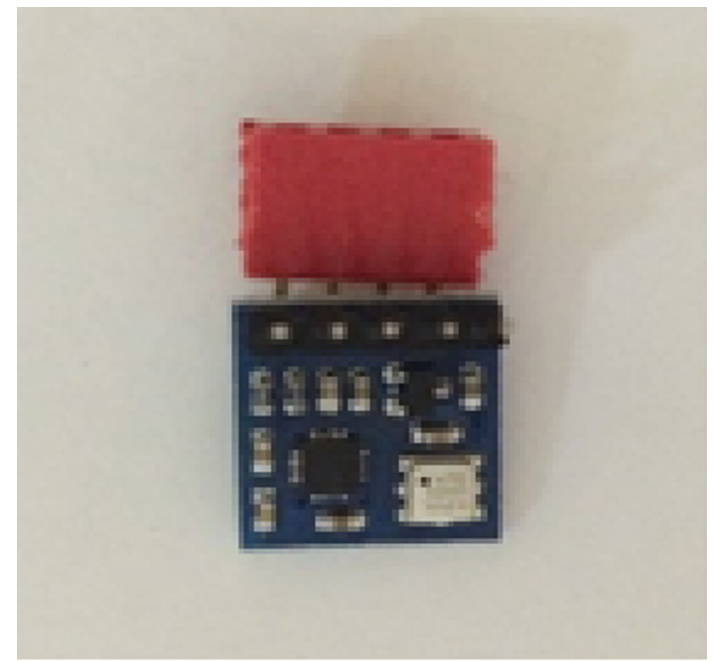

Figure 1. HMC9583L magnetic sensor integrated circuit.

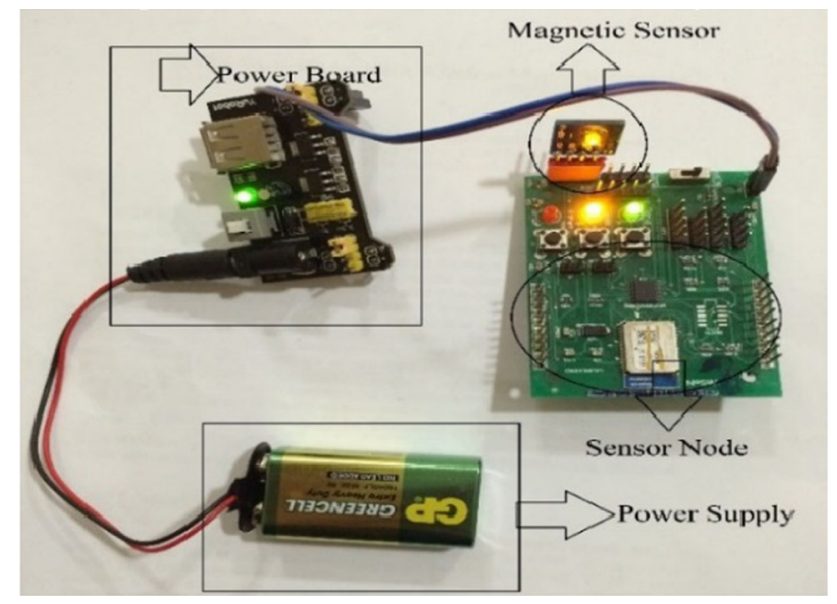

Figure 2. A sensor circuit designed for vehicle detection.

in terms of both size and cost; accordingly, anisotropic magnetoresistive sensors (AMRs) have been developed that can optimally detect the magnetic field of the Earth [7, 27].

Apart from containing 16 pins in its inner structure, an HMC9583L sensor (figure 1) has four usable pins on the outside. These are SDA/SPI_SDI, SCL/SPI_SCK, GND and VDD (2.16 v-3.6 v) pins (figure 2(b)). The GND pin is for grounding and the VDD pin is to establish a power connection. To operate the sensor at $3.3 \mathrm{v}$ through VDD, a power board circuit and a power supply (AA battery) were used. The main reason for using this sensor in this study is the possibility of serial communication when connected to the sensor node via pins with an $I^{2} C$ port. Thus, magnetic information is recorded in the data registers ( $\mathrm{X}, \mathrm{Y}$ and $\mathrm{Z}$ ) in a binary mode with the processor of the sensor node after every clock period obtained via the SCL (Serial
Clock) pin. The measurement method of the magnetic sensor via configuration registers A and B was adjusted to normal measurement mode because the measurement is done on the rising side of every clock period and data in the registers are updating. The information in these recorders can be used if necessary. This information gathered in the registers is transmitted to the sensor node via the SDA (Serial Data) pin at a serially determined frequency $(128 \mathrm{~Hz})$. On the other hand, for the clock frequency, CRA4, CRA3 and CRA2 bits in register A were made logic " 1 ," " 0 " and " 0 ," respectively. In this way, both more precise data were obtained. The magnetic field for each of the three axes was obtained by the conversion of the 2-byte magnetic value at each data register, $\mathrm{X}, \mathrm{Y}$ and $\mathrm{Z}$, to its value in modulo 10. Finally, the resultant value (C) was calculated with the help of the following equation.

$$
\mathrm{C}=\sqrt{X^{2}+Y^{2}+Z^{2}}
$$

The $\mathrm{C}$ value increases when a vehicle gets close to the nodes because the vehicles contain copious amounts of iron, nickel and cobalt (ferrous mass) and they alter the background ferromagnetic field. The magnetic field of the Earth is approximately 500 mGauss by default and the measured value increases when a vehicle passes a magnetic sensor within $0.5-1 \mathrm{~m}$. If the $\mathrm{C}$ value is divided by 256 , the magnetic field is found in units of gauss. For example, if the C value is 280 , then the magnetic field at that point is 1.09 gauss. A magnetic change in each direction causes a change in C. During vehicle detection with an HMC5983L magnetometer, the $\mathrm{C}$ value is grounded. If a vehicle passes by a magnetic sensor placed on the side or in the middle of the road, the measured $\mathrm{C}$ value exceeds the threshold $\left(C_{\text {threshold }}\right)$ and it is understood that a vehicle is on the road. Hence, the vehicle is detected.

The new sensor circuit used throughout the study for vehicle detection systems alongside the power board and the battery can be seen in figure 2 and the circuit block diagram in figure 3 . The sensor node, which operates under the Zigbee IEEE 802.15.4 standard in the $2.4 \mathrm{GHz}$ ISM band and maintains small size data intermission, is a lowcost device and a very low-power-consuming wireless sensor node. The designed wireless sensor node is a PA/ LNA-based Zigbee SoC (system-on-chip) integrated CC2530 and CC2591 manufactured by Texas Instruments. It was empowered with an MSP430, Ultra-Low-Power microcontroller unit (MCU). The difference between this node and the others such as TelosB or micaZ is that its hardware components can be optionally assembled. On this sensor node, SHT11 temperature and moisture sensor and Electronically Erasable Programmable Read Only Memory (EEPROM) are present alongside the many connectors that can be helpful in studies. Additionally, it contains a UART1 connector, to which a Sim900 node can be connected in 


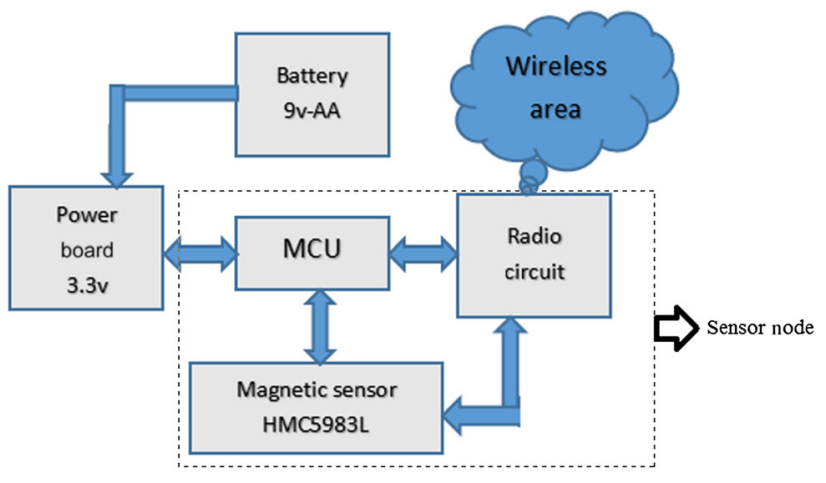

Figure 3. Block diagram of the sensor circuit.

order to transfer the collected data to the Internet and a UART2 connector to monitor the serial port output. As seen in figure 2, HMC5983L (magnetic sensor), which directly connects to the $I^{2} C$ port of the sensor node, can use the sensor as a compass along three axes. During the design of the sensor circuit, the 3.3-v output of the power board was connected to the VCC pin of the sensor circuit, as seen in figure 3. The angle with the magnetic poles of the Earth can be found using a 12-Bit Analog Digital Converter (ADC) with $1^{\circ}-2^{\circ}$ sensitivity.

Additionally, the sensor on the axes can communicate with the sensor nodes via the $I^{2} C$ protocol when a metal object gets close to the compass sensors. During the programming of the sensor, all the values on the sensor axes were read and the resultant value (C) was calculated using Eq. (1). If the sensor is used to just observe the effects of other objects on the magnetometer and not as a compass, then the resultant value will be enough. In this case, the resultant value, which is different at every place, changes when a metal object passes by. In this way, metal objects around the sensor can be identified.

\section{Experimental applications}

After the design of the multi-purpose sensor node and the circuit, three applications were performed as an experimental work and results were analysed using Matlab software. First of all, the four-level instantaneous traffic jam information of a single-lane road was obtained via vehicle detection by the magnetic sensor mounted on the sensor nodes. Then the vehicles passing by are classified in one of four categories: cars, minibuses, buses and trucks. Last, the direction of a passing vehicle was determined as being from left to right or from right to left.

\subsection{Determination of the traffic congestion}

In this part of the study, vehicle detection was due to the metal found on and in the vehicles. In this application, the wireless sensor network was constructed in order to detect the traffic condition on a $100-\mathrm{m}$ road with three sensor nodes placed as in figure 4. Each sensor node was programmed using $\mathrm{C}$ language with the Code Composer Studio open-source software. The three nodes used in the study were programmed as the end device (ED), whereas the other communal node was programmed as the coordinator. In fact, the coordinator node is not different from the other nodes; it just obtains the magnetic data simultaneously from the other three sensors due to the star topology of the design. Network setup starts when the coordinator node is turned on by means of the power board. There must be a coordinator node in every Zigbee network. During the programming of this node, PAN ID was assigned. Usually the assigned ID is 0x0000. This process reflects the identity of every network. When the Node 1, Node 2 and Node 3 sensors, which are all EDs, are turned on with the power board, the coordinator sends out a request to join the network for every predefined tm(measurement time).

The coordinator node was programmed according to a linear algorithm; in other words, the coordinator sends a simultaneous request to each ED node and waits for an answer for every $t m$ period, which can be interpreted by the blinking yellow light on the nodes. When the EDs join the network and send data to the coordinator, the yellow light glows. The coordinator node assigns a 16-bit Short Address to the ED that joins the network. This is the network address that distinguishes every node. ED nodes transmit the magnetic data collected from their surroundings to the coordinator node via RF (Radio Frequency) waves under the Zigbee communication standard; thus, the utilised network topology is a star topology. Because the coordinator node is connected to the computer with the programming interface via USB, the data acquired can be examined with the Tera Term serial port software. These data were imported to the Matlab workspace environment with the serial communication feature found in Matlab. Using the necessary parameters, the results were plotted in a graph.

4.1a Proposed algorithm for detection of the traffic congestion: In this study, an algorithm was proposed to determine the traffic congestion via vehicle detection. For this, three nodes were placed alongside the road at certain intervals and the results obtained were analysed. The resultant magnetic field value was calculated from the magnetic field values $M x, M y$ and $M z$ obtained by Node 1, Node 2 and Node 3 with HMC5983L using Eq. (1). In previous studies [7, 15], only $M x$ or $M z$ values were taken into consideration to detect vehicles. But the background magnetic field is a vector quantity in three dimensions, so in this study the resultant field from three axes is used. The algorithm to determine traffic conditions is defined in Algorithm 1. 


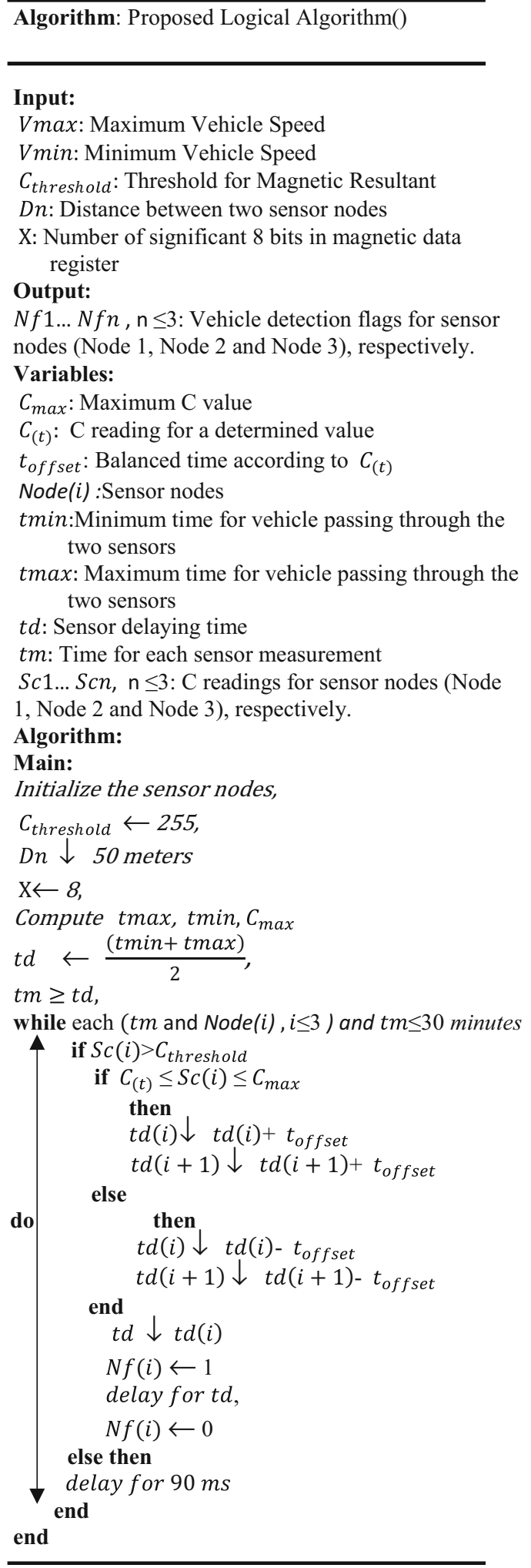

In the main part of Algorithm 1, sensor nodes are activated. In the study, $C_{\text {threshold }}$ was determined as 252 at first. But this value may change at different places, because the background magnetic field may be different for different places on Earth. Therefore, after many experimental applications, as seen in Algorithm 1, the $C_{\text {threshold }}$ value was updated to 255. It was assumed that for the values below 255 "there was no vehicle" and for the values above 255 "there was a vehicle," because there was the concern that the magnetic sensor may be affected by the other metal objects around. The important aspect here was that the information on existence of a vehicle was transmitted only for the $\mathrm{C}$ values above the threshold value. This means that the information acquired at the intervals when there was no vehicle detection was ignored. As seen in Algorithm 1, whenever a vehicle was detected by a node, detection flag $(N f(i))$ was fixed as " 1 " for $t d$ (delayingtime) seconds. After this time, $(N f(i))$ was assigned to " 0 ". Because a vehicle can only travel between the minimum and maximum speeds Vmin and $\operatorname{Vmax}(\mathrm{km} / \mathrm{h})$ it can travel the distance between any two nodes in a time interval between tmin and tmax (s). Therefore, $t d$ was taken to be between tmin and tmax. The $C_{(t)}$ value, which was defined to determine the adaptive $t d$ time, was considered to be low for the vehicles travelling at high speeds and high for the vehicles travelling at low speeds, because fast vehicles will have a shorter interaction time with the ground and, therefore, spends a shorter time in the coverage zone of the magnetic sensor with respect to slow vehicles. Therefore, the magnetic change induced by the vehicle that spends less time in the coverage zone will be smaller and its $\mathrm{C}$ value will be less than the one induced by a slow-travelling vehicle. According to Algorithm 1, if $S c(i)$ was bigger than a certain $C_{(t)}$ value, the equaliser time $\left(t_{\text {offset }}\right)$ was added to $t d$ time; otherwise, $t_{\text {offset }}$ time was subtracted from $t d$ time. Additionally, with $t d(i+1)$, the time for the next node to wait for was also adjusted. The measurement time of the sensor nodes, tm, must be bigger than or equal to $t d$, because it gives more accurate results to take another measurement after a longer time than the waiting period. In addition, it was understood from the experiments that the $\mathrm{C}$ value was also affected by the size of the vehicle. This was interpreted as larger vehicles, like a truck or a bus, contain a larger amount of metal. If the $\mathrm{C}$ value exceeded the threshold value $\left(C_{\text {threshold }}\right)$, the case of the vehicle being near the sensor was recorded as logic 1 . As it is understood, when any of the sensors detects a vehicle, this event is assigned as " 1 " by the program. When there is no vehicle detection, the value is " 0 ." The algorithm to determine the traffic congestion relies on the interpretation of the magnetic information coming from the sensors as " 1 " or " 0 ." Traffic density was evaluated in four categories. For example, if all the values coming from the sensors were " 1 ," then there is a "very heavy traffic" condition, and if all the values were " 0 ," then there is no traffic. Table 1 identifies all the possible traffic conditions. As seen in table 1 , if Node 1 gave " 0 ," Node 2 gave " 1 " and Node 3 gave "1," the traffic was considered heavy. On the other hand, since Node 1 was placed at the beginning of the road 


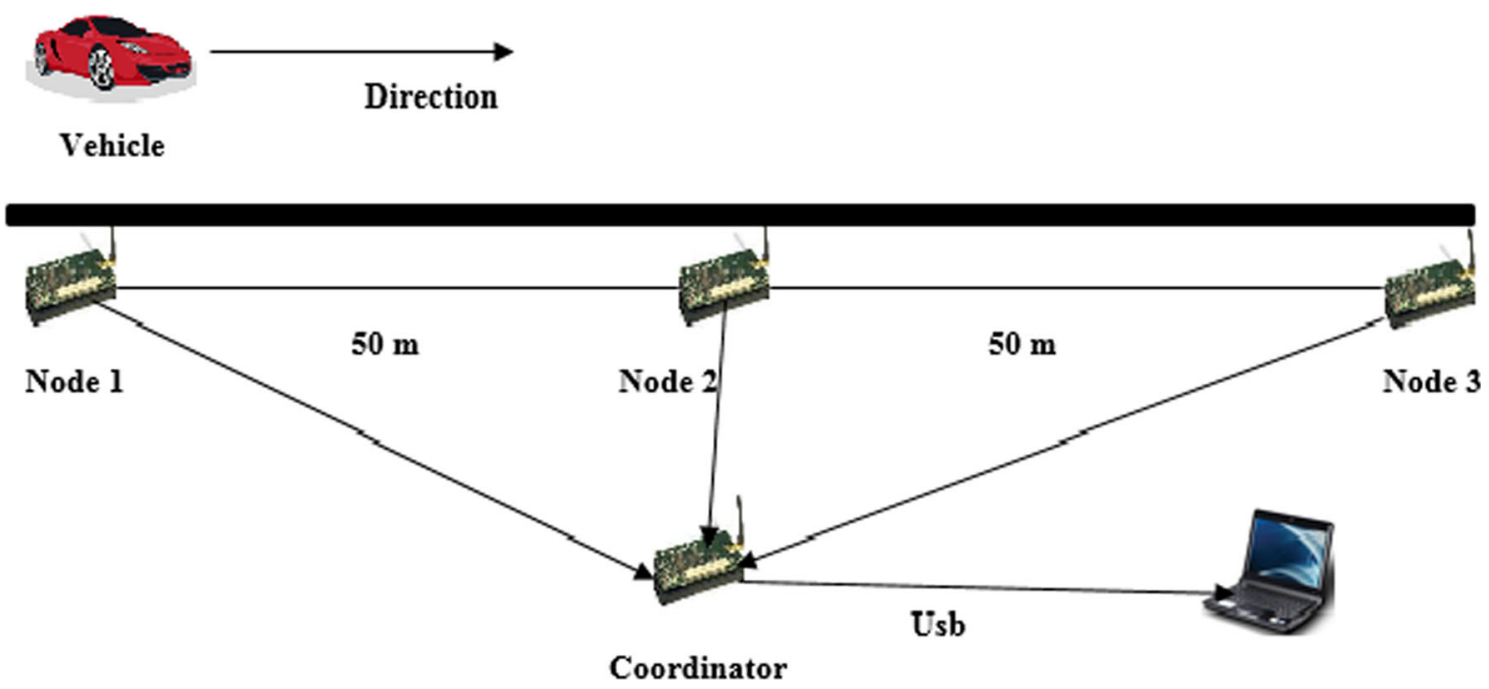

Figure 4. Wireless sensor network structure.

Table 1. Condition of the traffic according to the sensor data.

\begin{tabular}{lccc}
\hline Node 1 & Node 2 & Node 3 & State of traffic \\
\hline 0 & 0 & 0 & No traffic \\
0 & 0 & 1 & Mild traffic \\
0 & 1 & 0 & Mild traffic \\
0 & 1 & 1 & Heavy Traffic \\
1 & 0 & 0 & Mild traffic \\
1 & 0 & 1 & Heavy traffic \\
1 & 1 & 0 & Heavy traffic \\
1 & 1 & 1 & Very heavy traffic \\
\hline
\end{tabular}

and Node 3 at the end, a vehicle goes first by Node 1, then Node 2 and then reaches Node 3. Considering that the road is single lane and the vehicles have a $\operatorname{Vmax}=60 \mathrm{~km} / \mathrm{h}$, the minimum travel time between the two nodes, $50 \mathrm{~m}$ apart, is tmin $=3 \mathrm{~s}$. Additionally, for a minimum speed, $V \min =40 \mathrm{~km} / \mathrm{h}$, maximum travel time is tmax $=4.5 \mathrm{~s}$. Therefore, according to Algorithm $1, t d=3.75 \mathrm{~s}$. On the other hand, $t m$ was determined to be $4 \mathrm{~s}$, since it must be bigger than $t d$.

Figure 5 gives two images of the vehicle passing by the sensor. During the experiments, for the cases where $C_{(t)}$ had the limiting value $260, t_{\text {offset }}$ value was determined as 0.25 s. Hence, if $C_{(t)} \geq 260$, then $t_{\text {offset }}$ value was added to $t d$; otherwise, it was subtracted. For this application, the traffic condition was monitored for a 30-min period; the data to be examined was sent to Tera Term software via a serial port and then imported to Matlab software. But with a sampling logic, only the data between 14:20:25 and 14:20:56 are evaluated. Hence, the instantaneous traffic

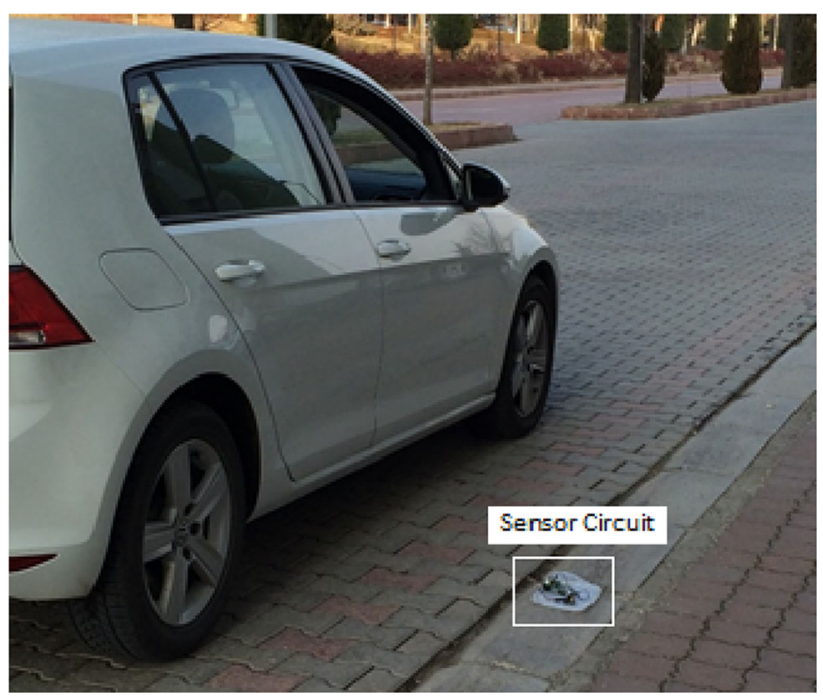

Figure 5. A vehicle approaching the sensor.

information was recorded for $31 \mathrm{~s}$. The data were plotted using Matlab software, where the time 14:20:25 was taken as the 0th and the time 14:20:56 as the 31st second. Figure 6 shows the information that came from the nodes for every second. As can be seen, for every second data coming from the nodes is " 0 " or " 1 ." For example, for the 0 th and the 1 st seconds, $\mathrm{C}$ value from Node 1, Node 2 and Node 3 was $252\left(252<C_{\text {threshold }}\right)$ and it was determined that there was no traffic due to logic value being " 0 ." But at the 2 nd second, a vehicle entered the road and the value measured by Node 1 became 258 and a logic " 1 " was sent. Then this 

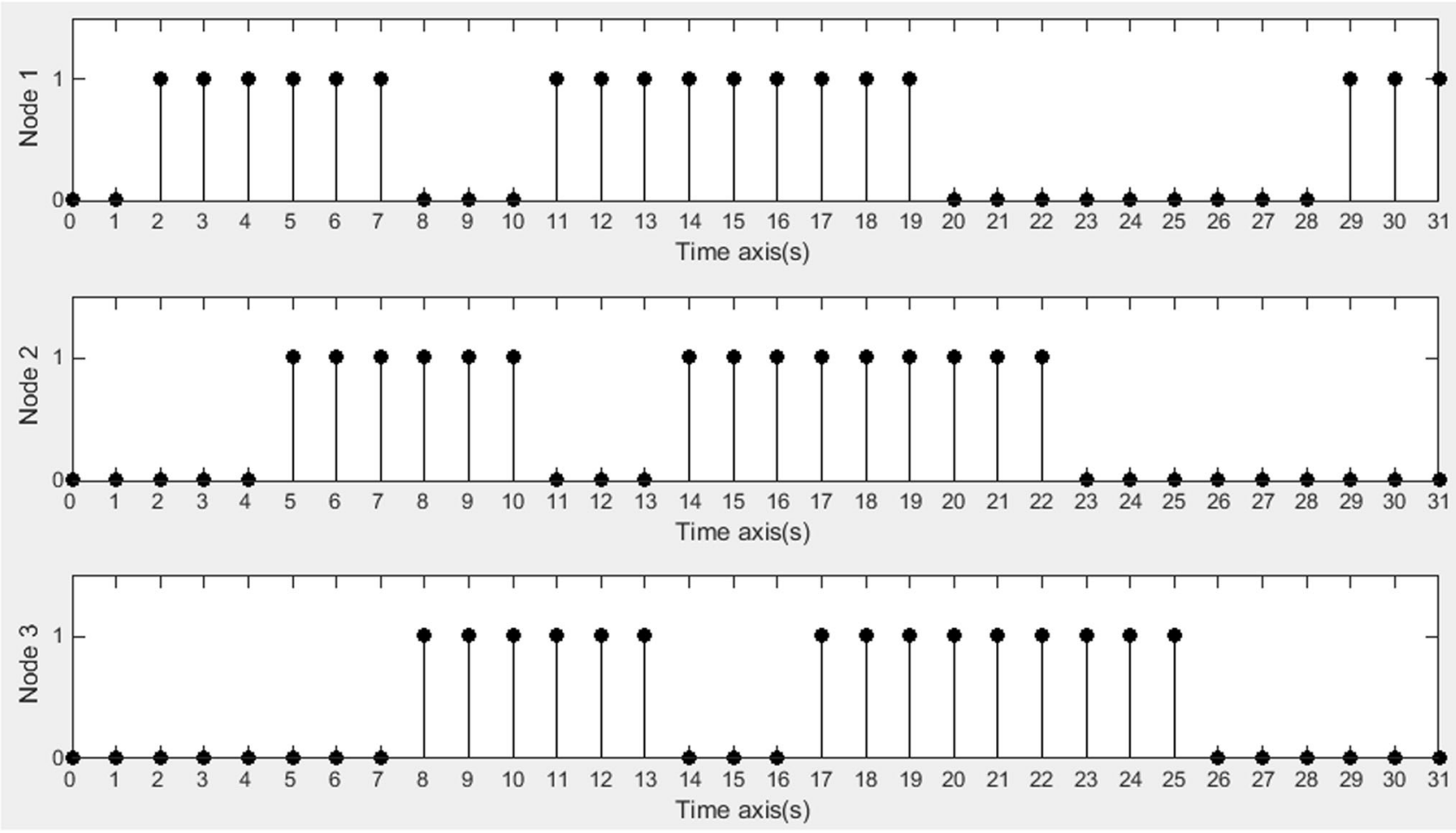

Figure 6. Values coming from the nodes.

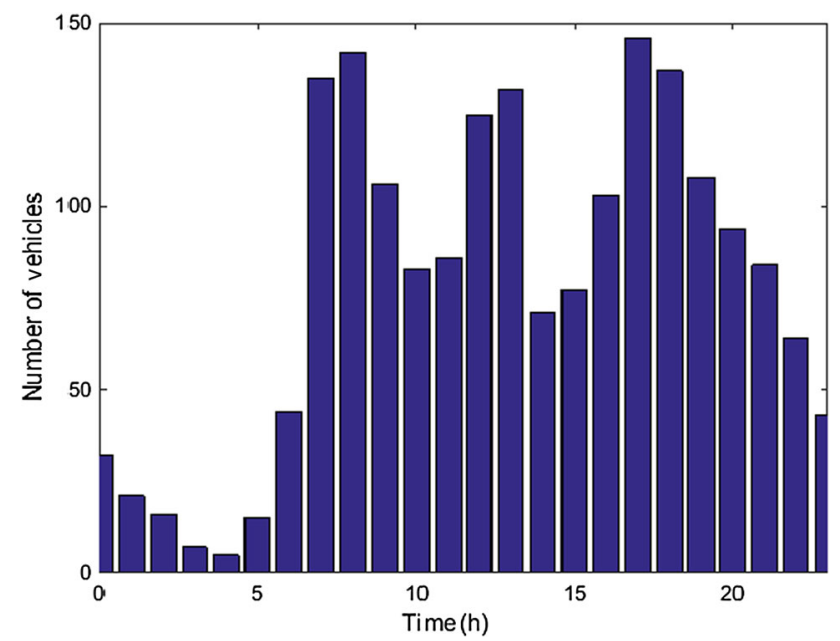

Figure 7. Number of vehicles-time plot with hourly intervals.

vehicle came close to Node 2 at the 5th second. At the 6th second, $\mathrm{C}$ value from the Node 1 was 259 and the logic value sent was " 1 "; $C$ value from the Node 2 was 258 and the logic value was also " 1 " and the $\mathrm{C}$ value from the Node 3 was 252 and the logic value was " 0 " and, therefore, traffic was categorised as heavy. As seen in figure 6 , the traffic was very heavy between the 17th and the 19th seconds, because the $\mathrm{C}$ values from the sensor nodes were 259 , 258 and 258 and logic value was "1" for each of them.
Between the 26th and 28th seconds there was no traffic, because all the values coming from the sensors were " 0 "; hence, there were no vehicles on the road.

As another application for this part of the study, the vehicles passing by Node 3 at the end of the $100-\mathrm{m}$ road were monitored every hour. The results were plotted using Matlab software where the time 00:00 was taken as the 0th hour and the time 23:00 as the 23rd. As can be seen in figure 10 , the number of vehicles passing by between the 17th and the 18th hours was much higher (135) than during the other time slots, and therefore, traffic was considered heavy. In addition, the number of vehicles passing by between the 4th and the 5th hours is very much lower than during the other time slots and therefore the traffic is considered mild. On the other hand, according to figure 7 , because the 7 th, the 8 th and the 9th hours are morning working hours, the traffic density was high. Compared with the other time slots, during the $3 \mathrm{rd}$, the 4 th and the 5 th, the traffic was mild, because these are night hours. These results can be shared with the drivers over the Internet if a server is setup.

\subsection{Vehicle classification}

In this section, different from the studies by Haijian, L., Honghui et al and Lifu et al [11, 12], the types of the vehicles were determined in four categories: cars, 


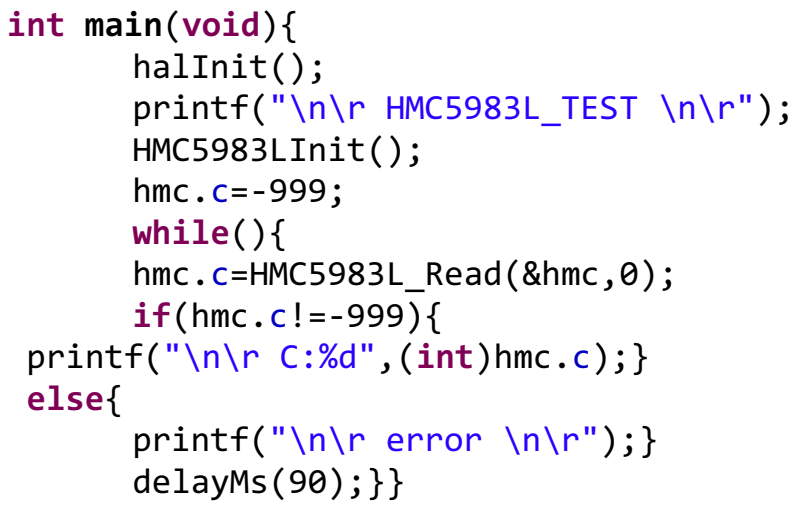

Figure 8. The code piece written in the Code Composer for the magnetic sensor.

minibuses, buses and trucks. To make this detection, a more precise and accurate measurement was needed and, therefore, an additional wireless sensor node was placed in the
Table 2. MSL limiting values determined for the vehicle types.

\begin{tabular}{lc}
\hline Limit value for vehicle types & $M S L_{\text {average }}$ \\
\hline$l_{c}$ & 3.5 \\
$l_{m}$ & 7.5 \\
$l_{b}$ & 14 \\
$l_{t}$ & 24 \\
\hline
\end{tabular}

middle of the road. In this application, again, the resultant magnetic field (C) was calculated using Eq. (1). A magnetic sensor was connected to the $I^{2} C$ port of the sensor node as in the previous applications. The sensor circuit, which may be considered an end node, transmits the magnetic data to the node that was programmed as the communal node. The ED converts the magnetic information in three axes ( $x$, $y$ and $z$ ) to the resultant magnetic field using a program that we wrote and transmits the value to the communal node

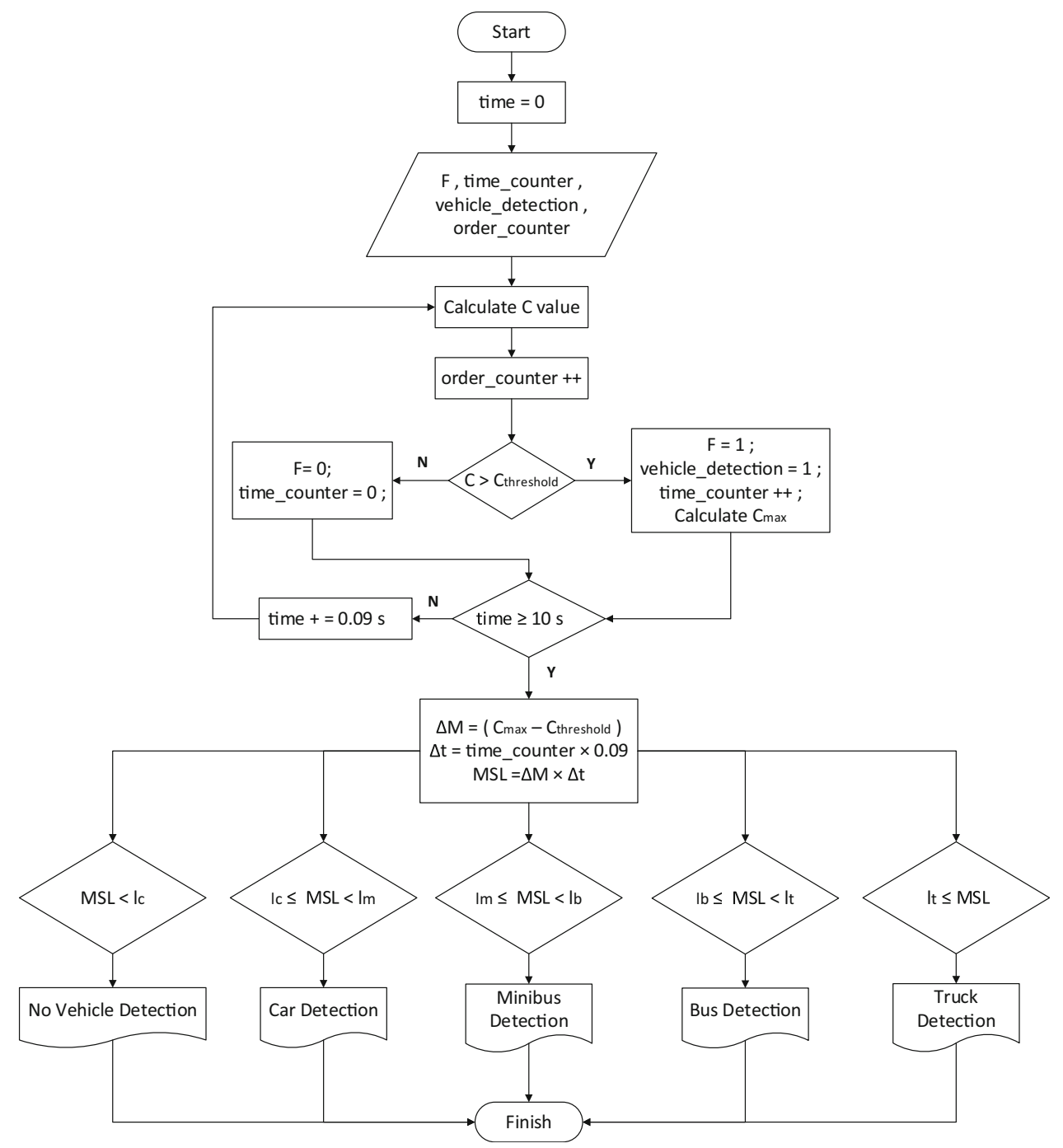

Figure 9. The flow diagram of the algorithm proposed for the vehicle classification (classification algorithm). 
every $90 \mathrm{~ms}$. Hence, the communal node sends the $\mathrm{C}$ value for every $0.09 \mathrm{~s}$ to the Tera Term terminal, which has serial port software. An HMC5983L magnetic sensor transmits the data obtained to the communal node every $90 \mathrm{~ms}$. In figure 8 , the code piece written in the hardware (hw.c) of the magnetic sensor is shown.

Reducing this time interval will increase the sample size of the magnetic data. Although an increase in the sample size will deliver more significant and sensitive results, Tera Term will not be able to obtain the data in that short time interval. Therefore, the sampling time was determined as $90 \mathrm{~ms}$, optimally.

In this application, the magnetic signature length $(M S L)$ was identified to classify the vehicles. $M S L$ can be calculated with the help of the magnetic amplitude difference $(\Delta M)$ and the occupation time $(\Delta t)$ as in the following equation:

$$
M S L=\Delta M \times \Delta t
$$

The magnetic amplitude difference is the difference between the measured resultant field and the predetermined threshold value. Occupation time, on the other hand, is the time the vehicle stays in the coverage zone of the sensor circuit. It is the product of the value given by the time counter defined in the sensor code with $90 \mathrm{~ms}$, because the delay measurement method is adjusted to $90 \mathrm{~ms}$. Depending on its $M S L$ size, the vehicle type can be determined as an automobile, a minibus, a bus or a truck. This depends on the length and the mass of the metals it contains. Figure 9 shows the flow diagram of the algorithm proposed for vehicle classification. One hundred vehicles consisting of equal numbers of automobiles, minibuses, buses and truck were made to pass by the sensors on the road in a random order and the minimum limiting $M S L$ values, $l_{c}, l_{m}, l_{b}$ and $l_{t}$ (figure 9) for cars, minibuses, buses and trucks,

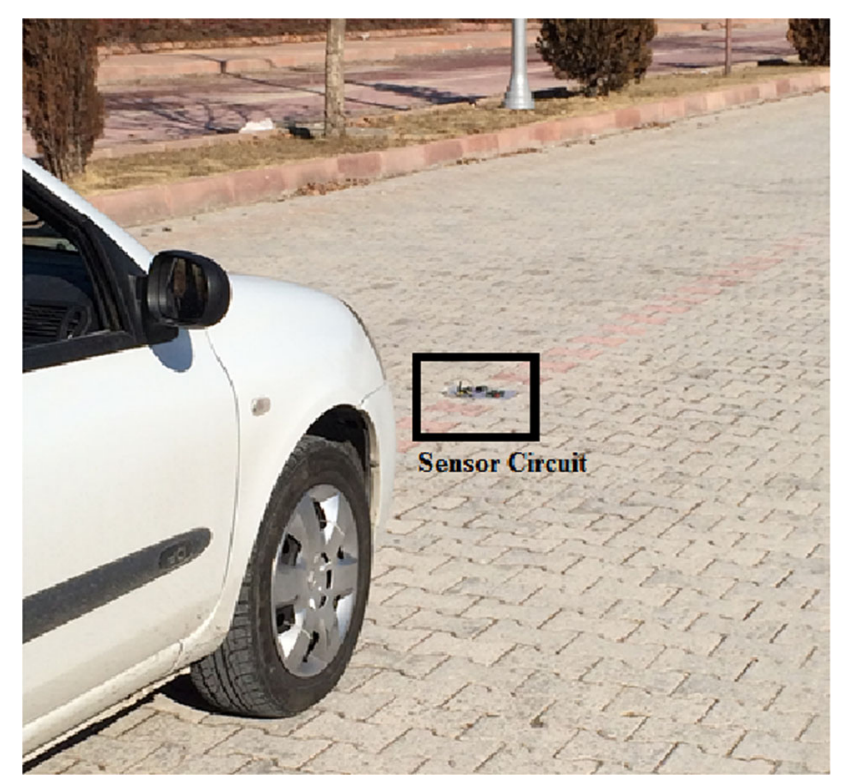

Figure 10. The car approaching the sensor circuit.

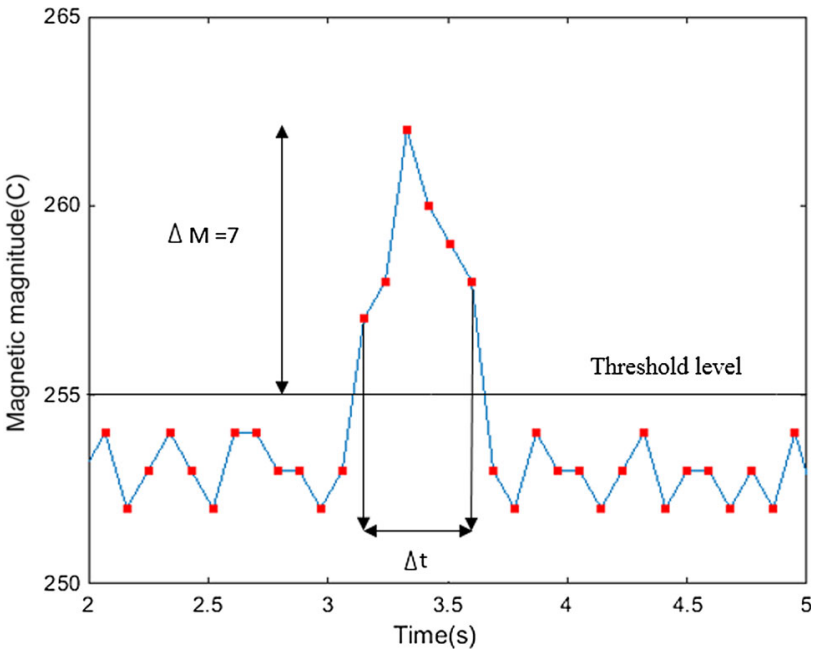

Figure 11. Resultant magnetic field-time plot (for car)

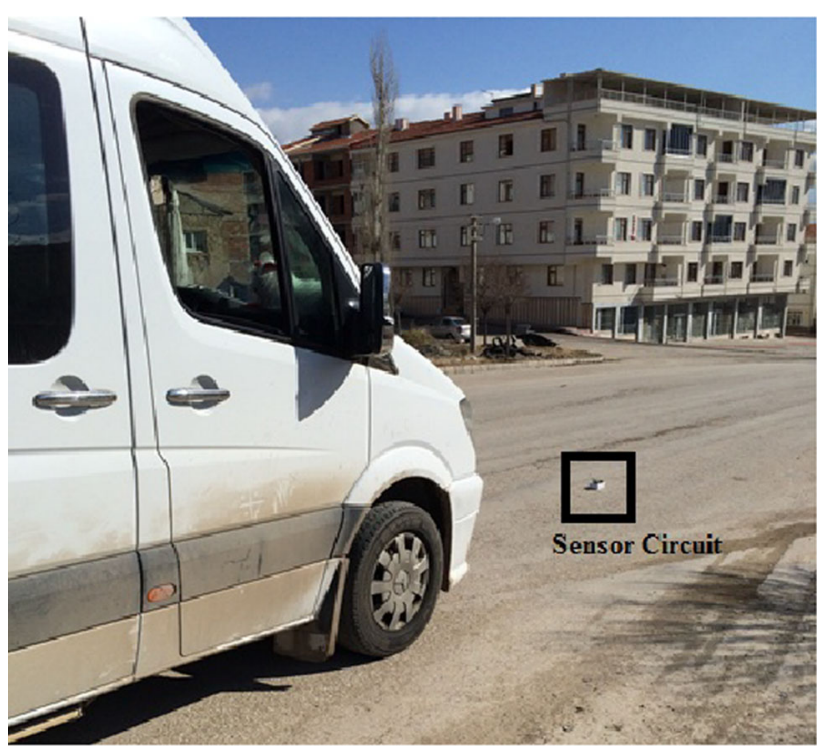

Figure 12. The minibus approaching the sensor circuit.

respectively, are shown in table 2 . For example, $l_{c}$ is the measured minimum $M S L$ value for cars. On the other hand, $l_{m}$ is both the maximum $M S L$ value for cars and the minimum $M S L$ value for minibuses. The important parameters for determining these values were the coverage distance between 0.5 and $0.75 \mathrm{~m}$, the differences in length of different types of vehicles and the mass of the metal the vehicle contains.

As seen in figure 9, if the $\mathrm{C}$ value was bigger than the $C_{\text {threshold }}$, then a vehicle was detected and the value of the time counter was increased by 1 . The flag value $(\mathrm{F})$ that declares the vehicle detection is coded as "1." The ordernumber parameter gives the number of measurements made. The type of the vehicle can be determined with the limiting values of $M S L$ given in table 2 . 


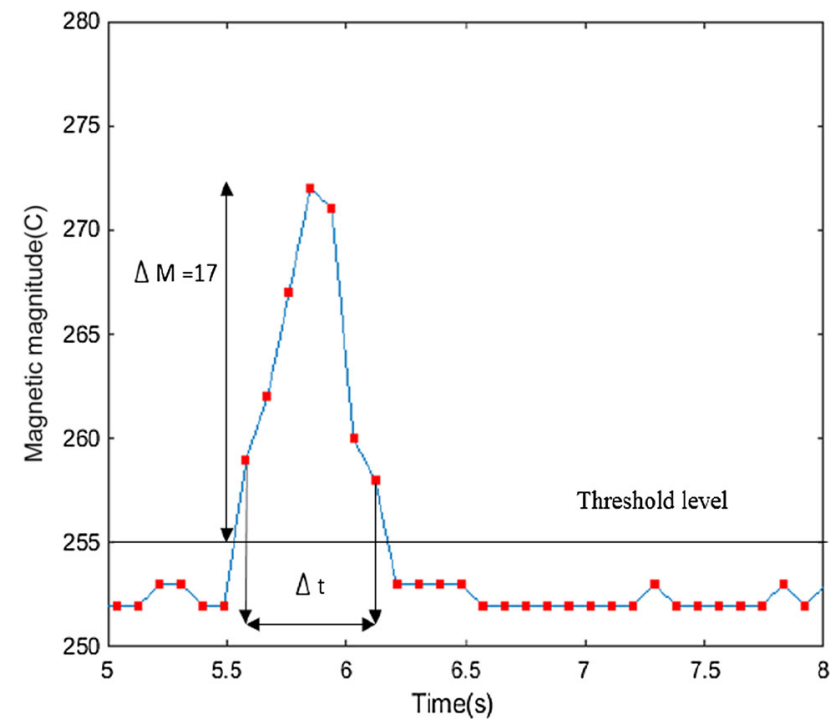

Figure 13. Resultant magnetic field-time plot (for minibus).

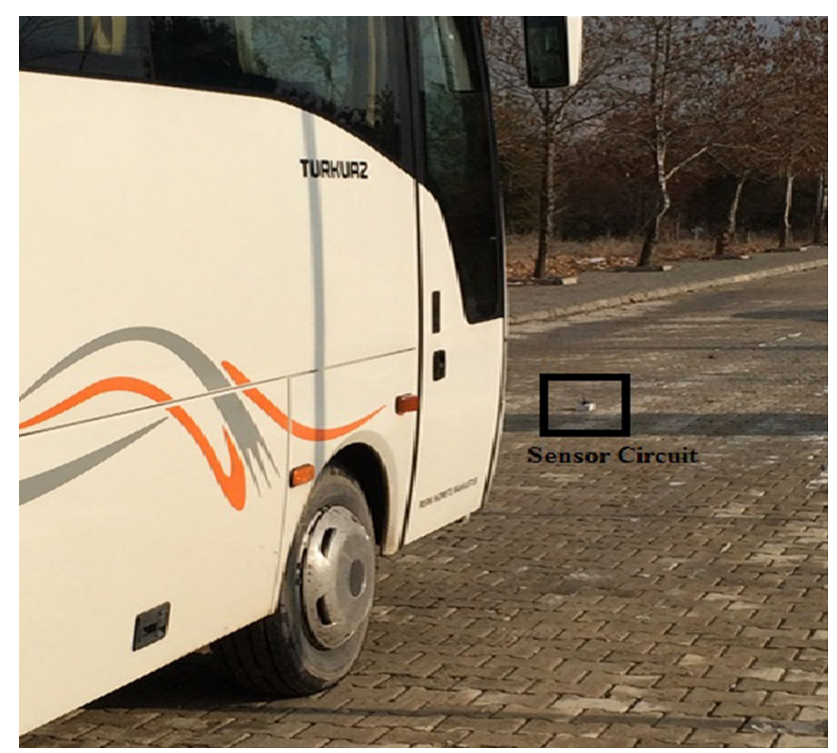

Figure 14. The bus approaching the sensor circuit.

In this application, first, a car was made to pass by the road at a constant speed of $40 \mathrm{~km} / \mathrm{s}$. It is known that a usual car can have a length from $3.6 \mathrm{~m}$ to $5.0 \mathrm{~m}$. Figure 10 shows the car passing by the road.

Because the data were stored in the log part of the Tera Term, only $\mathrm{C}$ values could be extracted by the data extraction method of Matlab software. Those values were examined with the Tera Term program every $0.09 \mathrm{~s}$ in a 10 $s$ period and the power boards of the sensors were shut down to end the measurements. These values are also plotted in figure 11. To clearly distinguish the detection time interval and the $C$ values, only values between the 2 nd

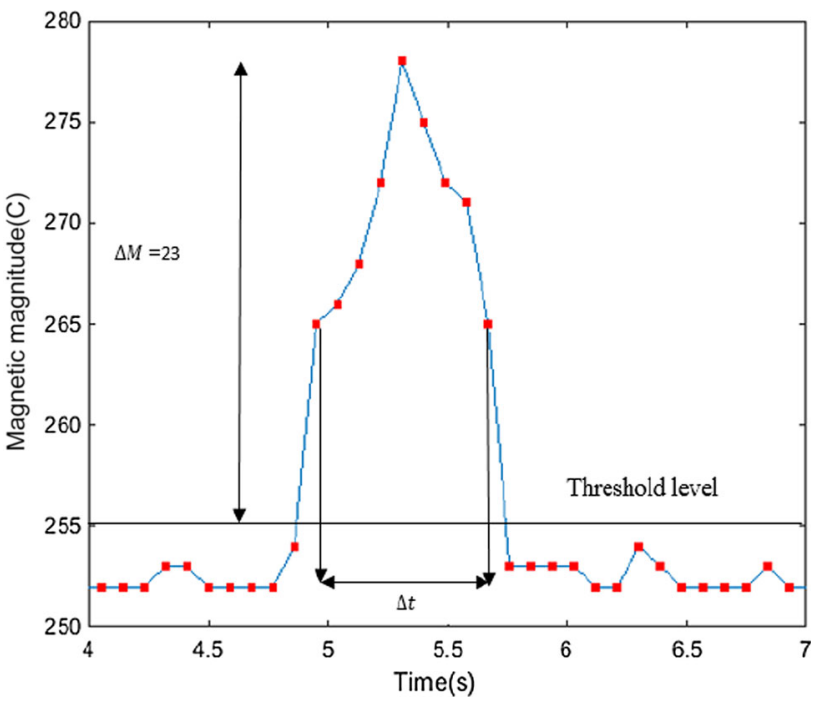

Figure 15. Resultant magnetic field-time plot (for bus).

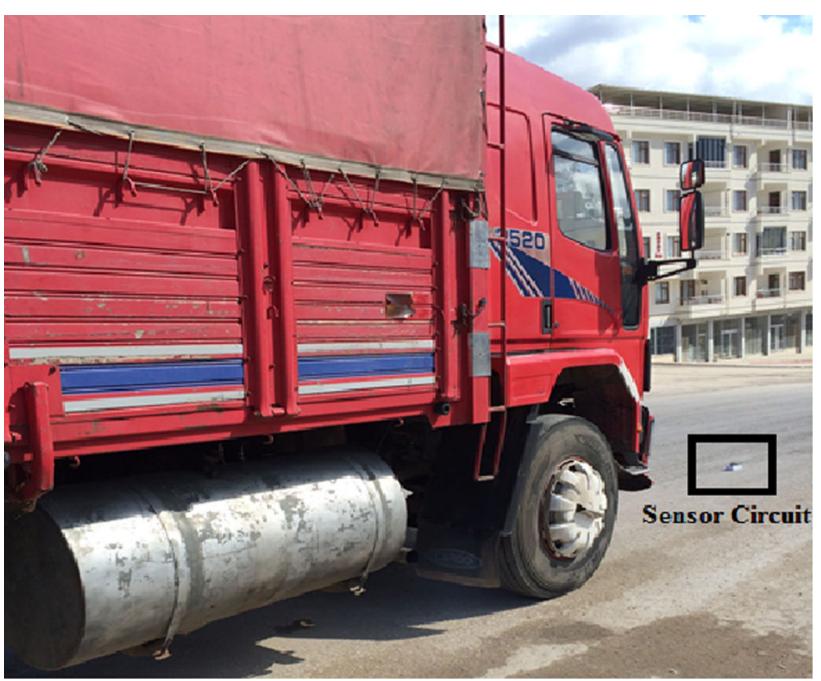

Figure 16. A truck approaching the sensor circuit.

and the 5th seconds are plotted. As shown in figure 11, during the time interval $\Delta t$, vehicle detection was recorded for six samples, meaning that the time-counter parameter had the value 6 . Because samples were taken once in $90 \mathrm{~ms}$, the time interval can be found as $\Delta t=$ $0.09 \times 6=0.54 \mathrm{~s}$. In addition, since the highest $\mathrm{C}$ value for a passing vehicle was 262 and the $C_{\text {threshold }}$ was 255 , magnetic amplitude difference can be calculated as $\Delta M=262-255=7$.

Thus, the magnetic signature length $(M S L)$ for this car could be found from Eq. (2), which gives 3.78. This value is between $l_{c}$ and $l_{m}$ according to figure 9 and therefore the detected vehicle was a car. The unit of the calculated $M S L$ value is of no importance; it just increases for minibuses or buses. 


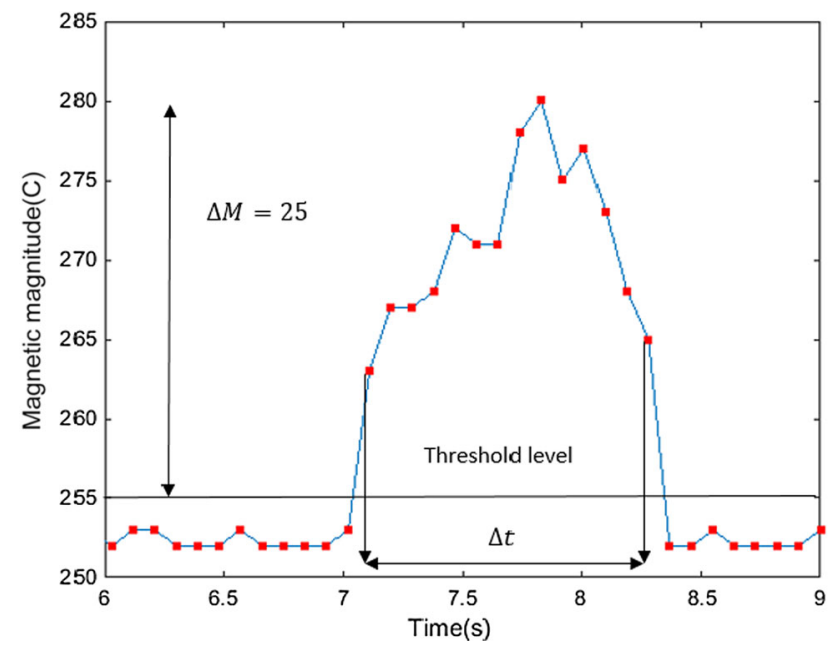

Figure 17. Resultant magnetic field-time plot (for truck).

Table 3. The average $M S L$ values for every vehicle type.

\begin{tabular}{lc}
\hline Vehicle type & $M S L_{\text {average }}$ \\
\hline Car & 5.23 \\
Minibus & 12.64 \\
Bus & 21.32 \\
Truck & 28.86 \\
\hline
\end{tabular}

As a second application, measurements were made for a minibus. Figure 12 shows the minibus approaching the sensor circuit. Because the length of the minibus is comparable to that of the car, the occupation times during passing by the sensor circuit were similar. But a minibus contains more metal and therefore its magnetic amplitude difference was higher than that of the car. Figure 13 shows the results plotted using Matlab software.

Again, to clearly distinguish the detection time interval and the $\mathrm{C}$ values, only values between the 5 th and the 8th seconds are plotted. As seen in figure 13, during the time interval $\Delta t$, vehicle detection was recorded for seven samples. This means the time-counter parameter had the value 7. Because samples were taken once every $90 \mathrm{~ms}$ and

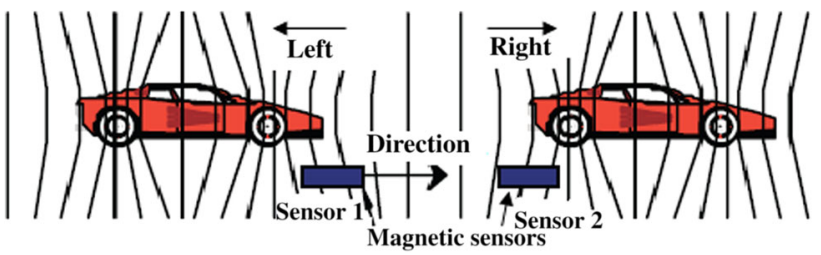

Figure 18. Direction determination scenario.

the highest $\mathrm{C}$ value for a passing by minibus was 272 and the $C_{\text {threshold }}$ was 255 , the magnetic signature length $(M S L)$ for the minibus can be found from Eq. (2), which gives 10.71. This value is between $l_{m}$ and $l_{b}$ according to figure 9 and therefore the detected vehicle was a minibus.

The third application was the detection of a city bus. These buses are about 7-9 $\mathrm{m}$ long, so their occupation time was longer than those of the cars or the minibuses. Figure 14 shows the bus approaching the sensor circuit. Figure 15 shows the data plotted for the bus. It can be seen from the plot that the time-counter parameter was 9. Hence, during the occupation time, nine samples measurements were taken.

To clearly distinguish the detection time interval and the $\mathrm{C}$ values, only values between the 4th and the 7th seconds were plotted. As seen in figure 15, during the time interval $\Delta t$ vehicle detection was recorded for nine samples. Because samples were taken once every $90 \mathrm{~ms}$ and the highest $\mathrm{C}$ value for a passing by bus was 278 and the $C_{\text {threshold }}$ was 255, the magnetic signature length $(M S L)$ for the bus can be found from Eq. (2), which gives 18.63. This value is between $l_{b}$ and $l_{t}$ according to figure 9 and therefore the detected vehicle was a bus.

Lastly, a truck was used for vehicle detection. Figure 16 shows a truck approaching the sensor circuit. During the application many measurements were taken. The most significant of the results are between 6th and 9th seconds and are plotted in figure 17. The most important result here is that the truck and the bus had similar magnetic amplitude differences but different occupation times. Because the detected truck was longer than the bus, its occupation time in the coverage zone of the sensor node is longer.

Table 4. Accuracies for the proposed vehicle classification algorithm.

\begin{tabular}{|c|c|c|c|c|c|c|}
\hline \multirow[b]{2}{*}{ Vehicle type } & \multirow[b]{2}{*}{ The number of vehicles passed } & \multicolumn{4}{|c|}{$\begin{array}{c}\text { The number of vehicles } \\
\text { detected }\end{array}$} & \multirow[b]{2}{*}{ Accuracy of the proposed algorithm $(\%)$} \\
\hline & & Car & Minibus & Bus & Truck & \\
\hline Car & 25 & 24 & 1 & 0 & 0 & 96 \\
\hline Minibus & 25 & 1 & 23 & 1 & 0 & 92 \\
\hline Bus & 25 & 0 & 1 & 23 & 1 & 92 \\
\hline Truck & 25 & 0 & 0 & 0 & 25 & 100 \\
\hline \multirow[t]{2}{*}{ Total number of vehicles } & 100 & 24 & 23 & 23 & 25 & 95 \\
\hline & \multicolumn{6}{|c|}{95} \\
\hline
\end{tabular}




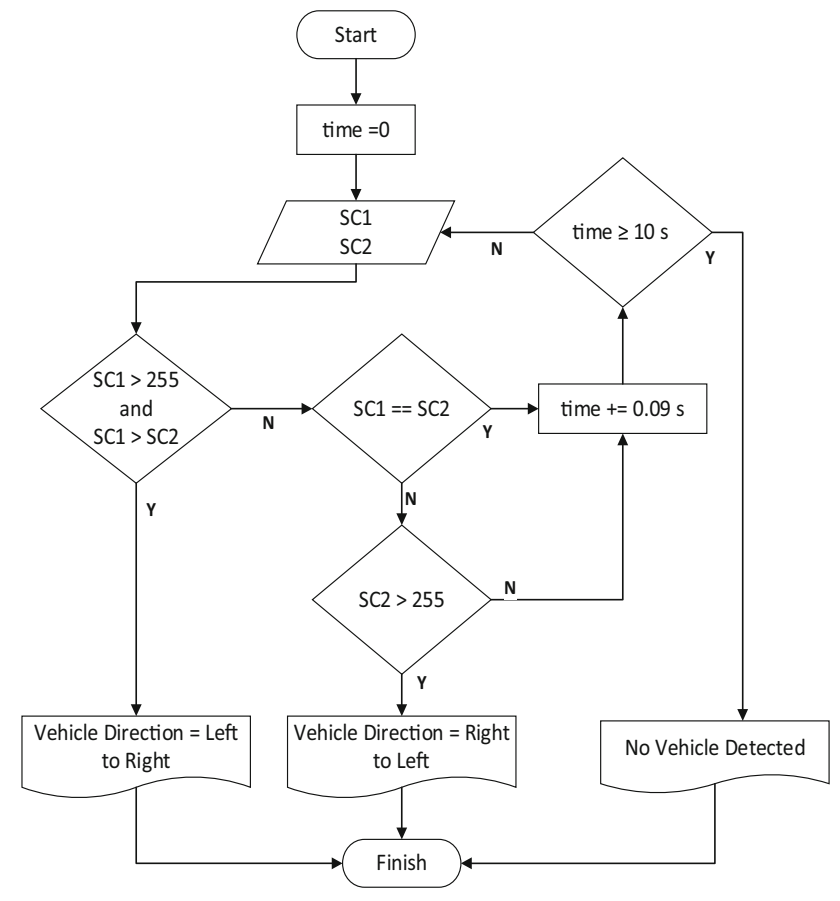

Figure 19. Flow diagram of the algorithm proposed to determine the vehicle direction.

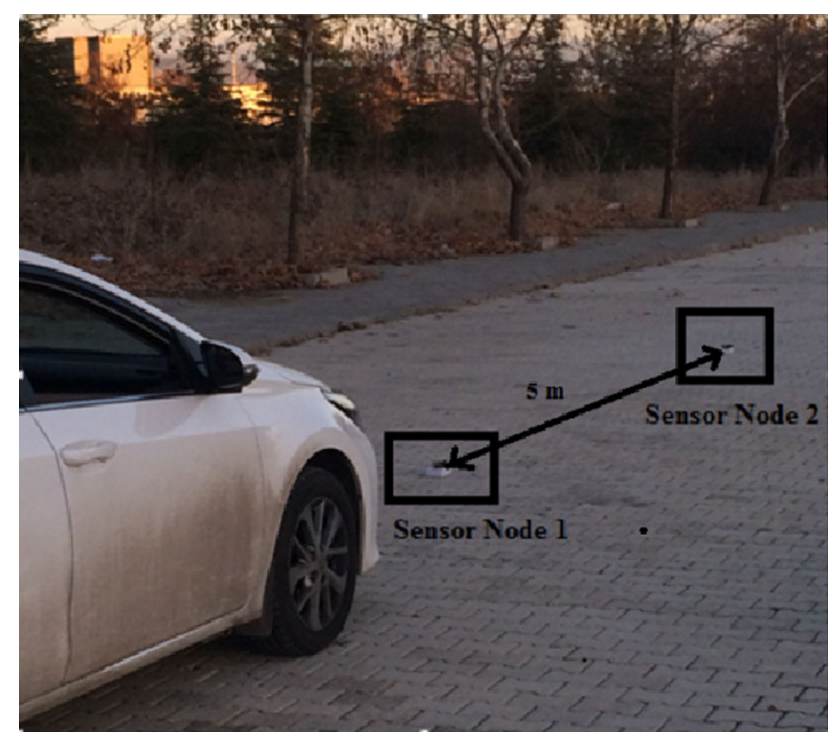

Figure 20. The vehicle approaching the sensor circuit.

As seen in figure 17 , during $\Delta t, 14$ samples of detection were taken and the results were recorded. The time-counter parameter was 14. Because samples were taken once every $90 \mathrm{~ms}$ and the highest $\mathrm{C}$ value for a passing truck was 280 and $C_{\text {threshold }}$ was 255 , the magnetic signature length (MSL) for the truck can be found from Eq. (2), which gives 31.5. This value is bigger than $l_{t}$ according to figure 9 and, therefore, the detected vehicle was a truck. Twenty-five

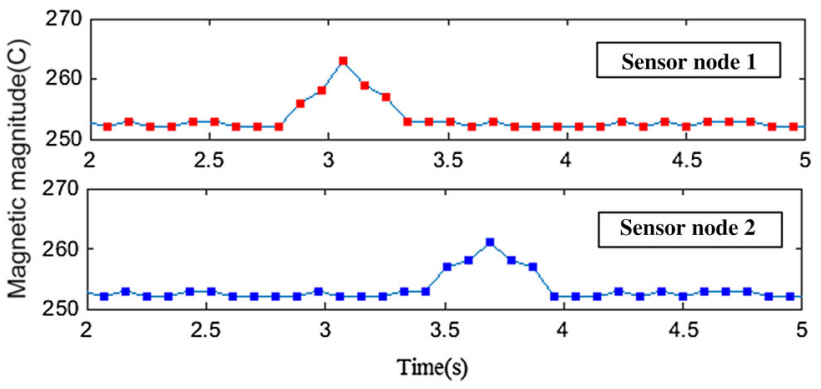

Figure 21. Determination of the vehicle direction, from left to right (resultant magnetic field (RMF)-time plot).

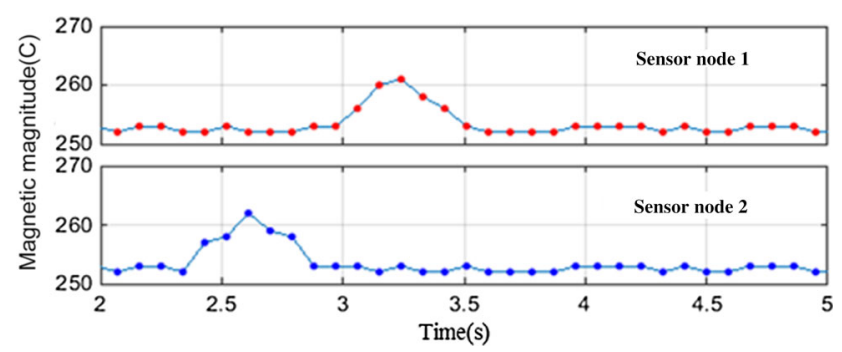

Figure 22. Determination of the vehicle direction, from right to left (resultant magnetic field (RMF)-time plot).

vehicles from each type passed along the road and the average $M S L$ values from the obtained data are summarised in table 3 . Table 4 shows the accuracy rates of the classification algorithm for 100 vehicles of different types. According to table 4, the highest accuracy was attained for trucks and the lowest accuracy for minibuses and buses, with $100 \%$ and $92 \%$, respectively. More significant, sensitive and accurate results were obtained for trucks because they are both large in size and rich in metal. On the other hand, if all vehicles are taken into account, the average accuracy is very high, with a rate of $95 \%$.

\subsection{Finding the direction of the vehicles}

In this section, the direction of motion of the vehicles passing by was determined. The direction of motion of the vehicles can be from left to right or from right to left. In this application, more than one sensor was used and the change in the background magnetic field due to the vehicles passing the sensors was measured in order to determine the direction of motion in one direction [28]. Figure 18 gives the application scenario for the determination of vehicle direction. In this application, to determine the direction of the vehicle, two sensor circuits were placed in the middle of the road $5 \mathrm{~m}$ apart. The aim here is to observe if the change in magnetic field was similar in the nodes placed nearby. The direction is determined when the resultant magnetic field $\mathrm{C}$ in one of the nodes exceeds the $C_{\text {threshold }}$ at a certain time before the other node detects the vehicle. 
Table 5. Accuracies for the proposed algorithm of vehicle direction determination.

\begin{tabular}{lcrcr}
\hline & & \multicolumn{2}{c}{ The number of vehicles } \\
detected & & \\
\cline { 3 - 4 } Direction of vehicle & The number of vehicles passed & Left to right & Right to left & Accuracy of the proposed algorithm (\%) \\
\hline Left to right & 28 & 26 & 2 & 92.9 \\
Right to left & 22 & 1 & 21 & 95.4 \\
Total number of vehicles & 50 & 26 & 21 & 94 \\
\hline
\end{tabular}

As in the previous application, the measurement time interval of the sensors was set to 90 . Figure 19 shows the flow diagram of the algorithm to determine the vehicle direction. SC1 and SC2 parameters in the diagram are the C values read at sensor 1 and sensor 2, respectively.

For this application, first, a car was made to pass by the sensor circuits at a constant speed of $40 \mathrm{~km} / \mathrm{s}$ as in figure 20. The direction on the $x$-axis is defined as from left to right if the car travels from sensor 1 to sensor 2 and from right to left otherwise.

Node 1 (sensor circuit 1) reported the existence of the vehicle in a setting where samples were taken every $90 \mathrm{~ms}$. Soon after, Node 2 (sensor circuit 2) also reported the vehicle. Hence, for a short period of time, Node 1 sent the logic value 1 and Node 2 sent the logic 0 . Then, Node 1 sent the logic 0 and Node 2 sent the logic 1 . These results were plotted with Matlab software and are shown in figure 21.

As seen in figure 21 , the vehicle was detected first by sensor circuit 1 and then by sensor circuit 2 . For example, the highest magnetic field value detected by sensor circuit 1 was 263 and by sensor circuit 2 was 261 . The occupation times in the coverage zone of the sensor were also similar.

Because a flow from sensor circuit 1 to sensor circuit 2 was seen, the direction of the vehicle was determined as from left to right. In addition to these, data acquired by the sensor were similar. Therefore, one can conclude that the same vehicle passed by the nodes.

The plot showing the vehicles travelling from right to left is given in figure 22. It can be seen that the vehicle was first detected by sensor node 2 and then sensor node 1 and therefore it is determined that the vehicle was travelling from right to left.

After that, a direction determination experiment was performed with 50 vehicles; of which, 28 were travelling from left to right and 22 were travelling from right to left. As seen in table 5, an accuracy of $92.9 \%$ was attained for vehicles travelling from left to right and $95.4 \%$ for the ones travelling from right to left. In general, the system has an accuracy of $94 \%$.

\section{Conclusion}

In this study, three applications of vehicle detection by the wireless magnetic sensors were developed and the results are evaluated and analyzed with various software tools. In the first of these applications, the traffic condition of $100 \mathrm{~m}$ of a single-lane road was analysed for $30 \mathrm{~min}$. In order to perform this application, a power board, a magnetic sensor and three sensor nodes with batteries were used. The aim was to examine the traffic on $100 \mathrm{~m}$ by placing three sensors $50 \mathrm{~m}$ apart. The traffic condition was classified with respect to the traffic density as no traffic, mild traffic, heavy traffic and very heavy traffic. With the obtained results, instantaneous traffic density could be observed at every second. In addition to these, with the information coming from Node 3 placed at the end of the road, the vehicles passing that section of road were counted all day and traffic was analyzed hourly. This way, traffic density information was determined with respect to the vehicles passing by per minute.

In the second application, the vehicles travelling on the road were categorised as four types: car, minibuses, buses and trucks. In this classification, an MSL parameter was proposed and used. As a result, the highest accuracy was attained in the truck classification with a rate of $100 \%$, whereas the lowest accuracy was attained for the minibuses and buses with a rate of $92 \%$. In this application, more significant, sensitive and accurate results were obtained for trucks because they are both big in size and rich in metal. But there were errors in differentiating minibuses and buses.

Lastly, the direction of the vehicles was determined as from left to right or from right to left. An accuracy of 92.9\% was obtained for the vehicles travelling from left to right and an accuracy of $95.4 \%$ was obtained for the ones moving right to left. The performance of the algorithm for the determination of the direction of vehicles was high and the general accuracy rate turned out to be $94 \%$. During the applications, many experiments were performed with the vehicles and samples were gathered from Tera Term software. It was seen that the proposed algorithm gave more accurate results with respect to the other studies.

These real-time results can be shared with the drivers in the traffic simultaneously. In this way, drivers can obtain information on the traffic condition (number, type and direction of the vehicles).

The most important features of this study were learning that the detection system is easy and more dynamic than the systems in other studies and that the proposed method and the algorithm deliver appropriate results. In addition, the number of hardware elements is few and low cost and the 
sensor nodes communicate with each other via Zigbee, a low-power-consuming communication standard.

But it will be more rational to design the sensor node, power board, magnetic sensor and the battery in a single integrated electronic medium. This way, problems arising when the circuit is placed on the road can be avoided. In addition, the sensor circuit can be protected from bad weather with a plastic fibre cover. With more sensor nodes, traffic on a longer road or on a road with two lanes can be monitored for a longer time.

\section{References}

[1] Haoui A, Kavaler R and Varaiya P 2008 Wireless magnetic sensors for traffic surveillance. Transp. Res. Part C: Emerg. Technol. 16(3): 294-306

[2] Lei Z, Wang R and Cui L 2011 Real-time traffic monitoring with magnetic sensor networks. J. Information Sci. Eng. 27(4): 1473-1486

[3] Gil Jimenez V P and Fernandez M J 2015 Simple design of wireless sensor networks for traffic jams avoidance. J. Sensor 2015: $1-7$

[4] Nooralahiyan A Y, Kirby H R and McKeown D 1998 Vehicle classification by acoustic signature. Math. Comput. Model. 27(9): 205-214

[5] Jo Y and Jung I 2014 Analysis of vehicle detection with wsnbased ultrasonic sensors. Sensors 14(8): 14050-14069.

[6] Leitloff J, Rosenbaum D, Kurz F, Meynberg O and Reinartz P 2014 An operational system for estimating road traffic information from aerial images. Remote Sensing 6(11): 11315-11341

[7] Michael J C and Withanawasam L S 1999 Vehicle detection and compass applications using AMR magnetic sensors. Honeywell, Plymouth, USA

[8] Chen W and Chen L 2005 A realtime dynamic traffic control system based on wireless sensor network. In: Proceedings of the 2005 International Conference on Parallel Processing Workshops (ICPPW'05). pp 258-264

[9] Nadeem T, Dashtinezhad S, Liao C and Iftode L 2004. TrafficView: a scalable traffic monitoring system. IEEE International Conference on Mobile Data Management (MDM'04), pp 1-14

[10] Ng E H, Tan S L and Guzman J G 2008 Road traffic monitoring using a wireless vehicle sensor network. International Symposium on Intelligent Signal Processing and Communication System. Bangkok, Thailand

[11] Haijian L, Honghui D, Limin J and Moyu R 2014 Vehicle classification with single multi-functional magnetic sensor and optimal MNS-based CART. Measurement 55: 142-152
[12] Lifu W, Nong Z and Haiping D 2015 Real-time identification of vehicle motion-modes using neural networks. Mech. Syst. Signal Process. 51: 632-645

[13] Haoui A, Kavaler R and Varaiya P 2008 Wireless magnetic sensors for traffic surveillance. Transp. Res. Part C: Emerg. Technol. 16(3): 294-306

[14] Sun C 2000 An investigation in the use of inductive loop signatures for vehicle classification. California PATH Research Report UCB-ITS-PRR-2002-4

[15] Cheung S Y, Coleri S, Dundar B, Ganesh S, Tan C W and Varaiya P 2004 Traffic measurement and vehicle classification with a single magnetic sensor. California PATH Research Report UCB-ITS-PWP-2004-7

[16] Vancin S and Erdem E 2015 Design and simulation of wireless sensor network topologies using ZigBee standard. Int. Comput. Netw. Appl. 2(3): 135-143

[17] Mihajlov B and Bogdanoski M 2011 Overview and analysis of the performances of Zigbee-based wireless sensor networks. Int. J. Comput. Appl. 29(12): 28-35

[18] Wang X and Zhang S 2010 Comparison of several sensor deployments in wireless sensor networks. International Conference on E-Health Networking, Digital Ecosystems and Technologies. pp. 236-239

[19] Gans J S, King S P and Wright J 2005 Wireless communications. Handbook of telecommunications economics. ISBN: 0444514236

[20] Chang K $2000 R F$ and microwave wireless systems. ISBNs: 0-471-35199-7, John Wiley \& Sons, Ltd

[21] Karasulu B, Toker L and Korukoğlu S 2009 ZigBee - IEEE 802.15.4 Standard based wireless sensor networks. International Conference, Information Üniversity, İstanbul, Turkey

[22] Callaway E, Gorday P and Bahl V 2002 Home networking with IEEE 802.15.4: a developing standard for low-rate wireless personal area networks. Commun. Mag. IEEE 40(8): 70-77

[23] Markevicius V, Navikas D, Daubaras A, Cepenas M, Zilys M and Andriukaitis D 2014 Vehicle influence on the earth's magnetic field changes. Res. J. Elektronika IR, Elektrotechnika 20(4): 43-48

[24] Ciureanu P and Middelhoek S 1992 Thin film resistive sensors, Institute of Physics Publishing, New York

[25] Jonasson C, Erlandsson M and Johansson C 2006 Magnetic sensors for traffic detection. IMEGO, Technical Report, Sweden

[26] Prateek G V, Rajkumar V, Nijil K and Hari K V S 2012 Classification of vehicles using magnetic dipole model. TENCON 2012-IEEE Region 10 Conference, Cebu, pp. 1-6

[27] Piecha J 2012 Digital camera as a data source of its solution in traffic control and management. Transp. Problems 7(4): $57-70$

[28] Sifuentes E, Casas O and Pallas-Areny R 2011 Wireless magnetic sensor node for vehicle detection with optical wake-up. IEEE Sensors J. 11: 1669-1676 